\title{
NEUROLEPTANALGESICS: 2. LABORATORY EVALUATION OF COMBINATION OF ANALGESICS AND NEUROLEPTICS WITH NITROUS OXIDE*
}

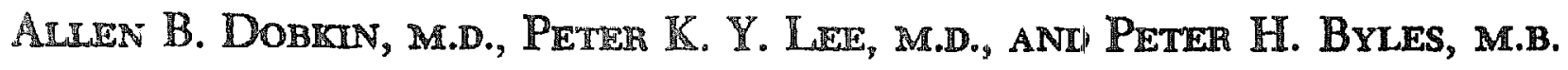

IN THE CONTINUING SEARCH for anaesthetic agents which produce the minimum disturbance of the normal physiology yet are clinically effective, a major impediment to a truly scientific approach, rather than empiricism, is the fact that there is still a serious lack of precise lonowledge of how quite minor changes in drug molecular structure may produce such profound changes in pharmacological action, and of how different agents interact with one another. ${ }^{1}$ However, we have found that the combination of certain drugs does complement our aim clinically and oftentimes reduces the undesirable effect of using a large dose of a single agent. ${ }^{2-4}$

Janssen recently introduced two groups of drugs.5,6 One, the butyrophenones, has neuro- and psycho-sedative properties similar to those of the phenothiazines. The other (meperidine-like) has analgesic properties of great potency, and was at first thought to be without the propensity to cause addiction, but now is known to be addictive. These have been reinvestigated in detail in America during the past two years. ${ }^{7-12}$ The expression "mineralized" has also been used to describe the state produced by injection of these drugs in combination into humans, ${ }^{13}$ and this state is felt by some to be appropriate to the needs of a surgical operation.

Recently, also, Harris and Pierson of Winthrop-Sterling Research Institute have studied a number of compounds that are in the benzomorphan series and are rather similar to nalorphine. ${ }^{14}$ Pentazocine (Win 20, 228) has about onethird the analgesic potency of morphine, whereas cyclazocine is at least five times as potent as morphine. The subjective effects of morphine and pentazocine appear to be similar, causing restlessness, itchiness, dizziness, grogginess, nausea, and talkativeness. Cyclazocine differs only in that it appears to be somewhat more likely to produce hallucinations. The respiratory depressant effect of both new compounds is felt to be similar to that produced by equianalgesic doses of morphine. Unfortunately, if respiratory depression is produced by these compounds, it is not antagonized by nalorphine. Cass and associates recently reported that pentazocine produces satisfactory pain relief of approximately 2 hours' duration with less side-effects than morphine. ${ }^{15}$ Lasagna has reported the same effect with cyclazocine. ${ }^{16}$ Of significant interest is that pentazocine appears to have no addicting properties, but this has been the unsubstantiated claim for every new analgesic except for methotrimeprazine. ${ }^{17}$

\footnotetext{
From the Department of Anesthesiology, State University of New York, Upstate Medical Center, Syracuse, New York, 13210. Presented at the Annual Meeting of the Canadian Anaesthetists' Society at Montebello, Quebec, Canada, in May 1964.
} 
Two well-known phenothiazine derivatives, methotrimeprazine (levomepromazine, Nozinan ${ }^{\oplus}$ ) and promazine (Sparine $\left.{ }^{(}\right)$, were included in this study because they are powerful neuroleptics. Methotrimeprazine has a wide range of properties that are useful in anaesthesia, including a purported powerful analgesic effect that is said to be equivalent to that of morphine.17-19 The properties of promazine are very similar to those of chlorpromazine, but it might be less likely to cause hypotension when given during anaesthesia. ${ }^{20}$

Chlorprothixene (Taractan ${ }^{\oplus}$, Tarasan $\left.{ }^{(}\right)$was used because it is also a potent neuroleptic belonging to the thioxanthene group of drugs, which is c'osely related in structure to the phenothiazines. In laboratory and clinical tests of this compound, we have found that it hardly differs from chlorpromazine in those properties that might be useful in anaesthesia. ${ }^{21-23}$

The pharmacology of the individual drugs used in this study is summarized in Figure 1.

The object of this study was to test various mixtures of these analgesic and neuroleptic drugs to determine whether they provide satisfactory anaesthetic conditions and to compare these with some mixtures which we have already tested in animals and in man. ${ }^{8,9,20,23}$

\section{Material and Methods}

Following preliminary experiments to devise appropriate drug mixtures, 12 combinations that appeared to be satisfactory were selected for controlled tests and were used for comparison with mixtures previously tested-thiopentalcurare, fentanyl-droperidol, and thiopental-methotrimeprazine. With these, 115 experiments of the crossover type were carried out on 30 dogs weighing approximately $20 \mathrm{~kg}$. (18 to $26 \mathrm{~kg}$.). Groups of 5 or 10 dogs were used depending on whether the experiment was proceeding well. Each animal was premedicated with atropine $0.5 \mathrm{mg}$. and scopolamine $0.5 \mathrm{mg}$. intramuscularly, an hour before injection of the anaesthetic drugs. An intravenous infusion of 0.9 per cent saline was started in a forepaw. Succinylcholine $20 \mathrm{mg}$. was injected and, after hyperventilation with oxygen, the trachea was intubated with a cuffed endotracheal tube lubricated with 4 per cent lidocaine ointment and the tube was connected to a Takaoka respirator. ${ }^{24}$ Pulmonary ventilation of each animal was set at $400 \mathrm{ml}$. gas $/ \mathrm{kg}$. body weight using a $2: 1$ mixture of nitrous oxide and oxygen. A Wright respirometer was interposed between the tube and the respirator to check and adjust the minute ventilation. ${ }^{25}$

With local infiltration anaesthesia ( 1.5 per cent lidocaine), plastic catheters were placed in the femoral artery and vein and threaded into the aorta and inferior vena cava in order to record blood pressures via Statham strain gauges. These were flushed as required with small amounts of heparinized saline. (No glucose was administered during any of the experiments.) After preliminary preparations were completed, the dog was placed in the lateral recumbent position on the laboratory table.

The rate of respiration was derived from the tracing using a belt-type pneumotachygraph. A rectal thermometer, urinary catheter with calibrated trap, 


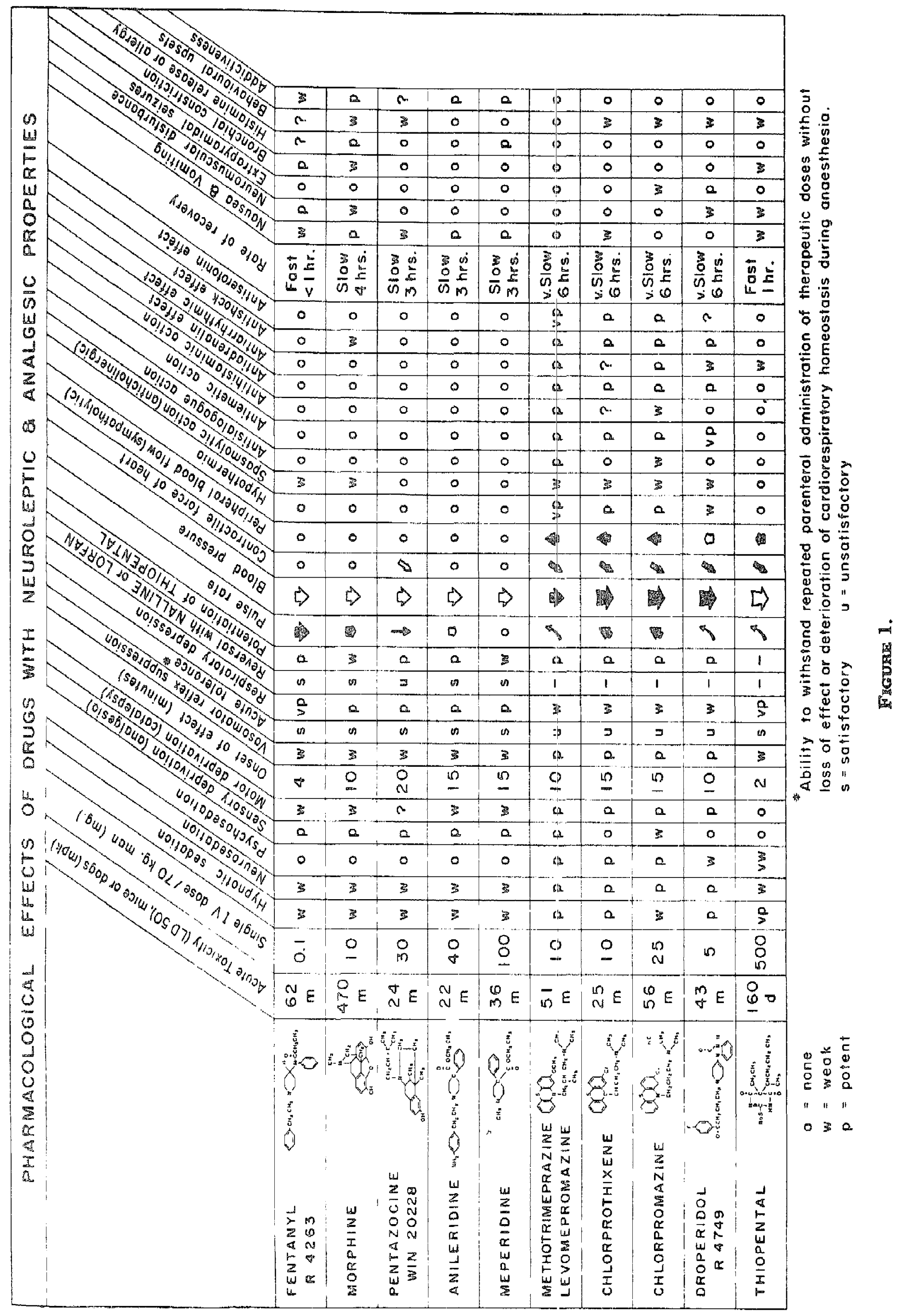


and lead 2 of the E.C.G. were attached to record body temperature, urine output, and cardiac rate and rhythm during the experiment. E.C.G., blood pressures, and respiration rate were recorded continuously and simultaneously throughout each experiment on a Grass polygraph ink recorder.

In each experiment, 20 per cent of the calculated dose was injected through the saline infusion at the beginning of the study and, after 10 minutes, the remainder was similarly injected. During preliminary experiments with some of the mixtures, it was found that the dose selected was not sufficient to provide smooth anaesthesia, so supplemental doses were given and the dose selected was revised during subsequent tests until a satisfactory one was reached and employed in the comparative experiments Sixty minutes after the full dose was administered, artificial respiration with nitrous oxide and oxygen was discontinued and 100 per cent oxygen was insufflated into the endotracheal tube. The hind paw was pinched at 5-minute intervals until the dog responded physically; then all recording apparatus was removed, the procedure was terminated, and the dog was placed on the laboratory floor. The animals were then kept under direct observation until they were fully recovered from the anaesthetic and were able to ambulate. The occurrence of retching, vomiting. defaecation, and rate of recovery were recorded.

Arterial blood samples were drawn at the beginning and end of each anaesthetic and again when the animals became responsive. These were analysed for $\mathrm{pH}, p \mathrm{CO}_{2}$, and $p \mathrm{O}_{2}$ using an Epsco Medical Blood Parameter Analyzer which uses a constant-temperature bath set at $37^{\circ} \mathrm{C}$., a Metrohm constant-temperature $\mathrm{pH}$ electrode, a Clark platinum silver membrane-covered electrode for measuring oxygen tension, and a carbon dioxide tension electrode. ${ }^{26-28}$ Venous blood samples were drawn before and at the end of anaesthesia to measure the haematocrit, blood sugar, blood urea nitrogen, serum potassium, SGOT, and SGPT. ${ }^{29-32}$

Digital tables were prepared showing a summary of the data from the tracings and laboratory procedures from each experiment. These were analysed for each group of anaesthetic tests and evaluated to determine the over-all efficacy of the anaesthetic mixture, and its probable value as a clinical anaesthetic technique.

\section{ResUlts}

Tables II to XV show the analysed serial effects of each drug mixture on vital signs. In Tables XVI to XXX, the corresponding effects on metabolism are shown. The data in the Tables I-XXX are summarized in Tables XXXI and XXXII to show the specific changes that were induced by each anaesthetic mixture, so that they can be compared easily.

As previously reported, the mixture of fentanyl and droperidol (Innovar ${ }^{(1)}$ ) and the mixture of methotrimeprazine and thiopental provide satisfactory anaesthetic conditions without causing any appreciable cardiovascular or metabolic upset. ${ }^{8}$ Similar satisfactory anaesthetic conditions were provided with all of the mixtures containing thiopental (with anileridine, fentanyl, meperidine, 


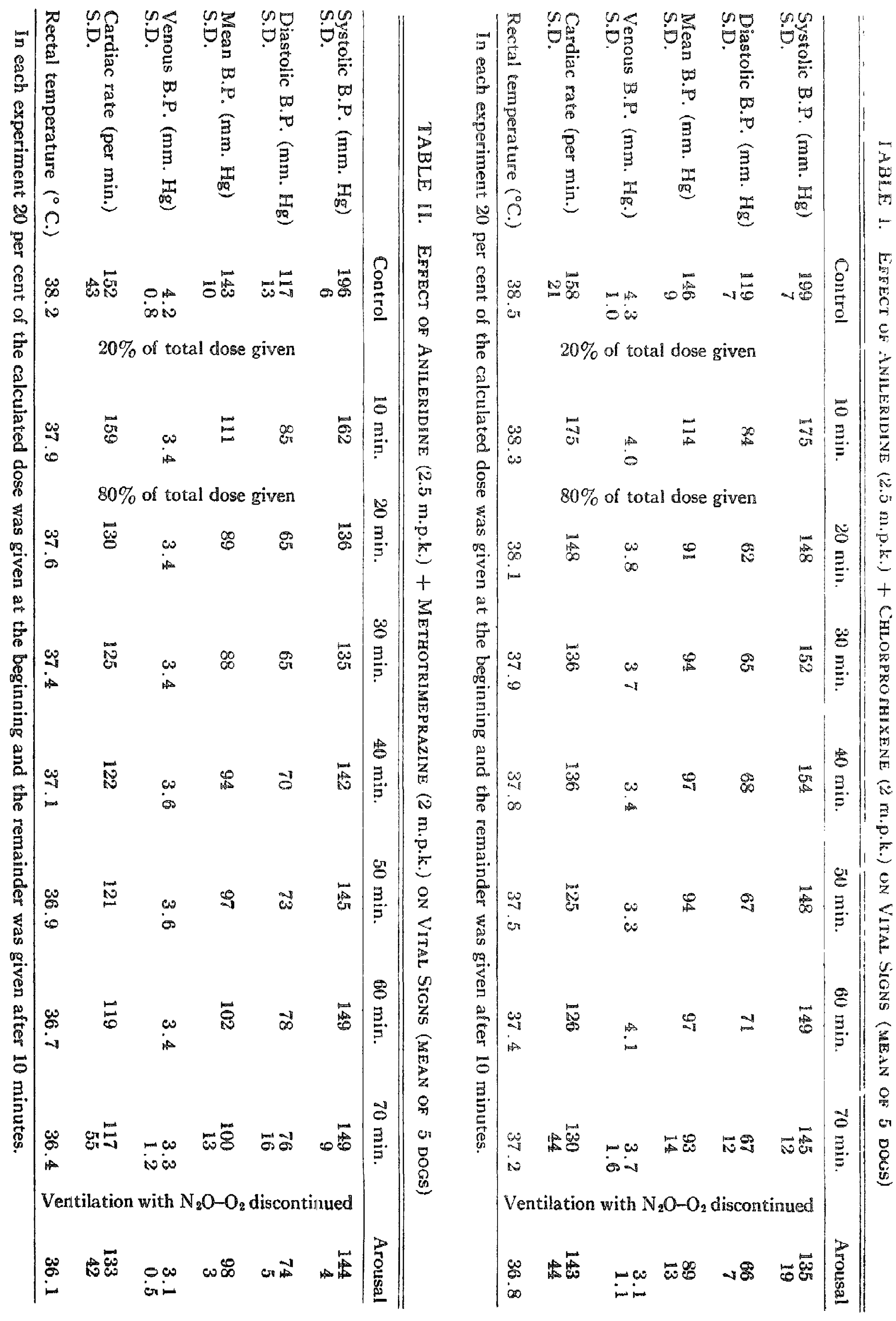




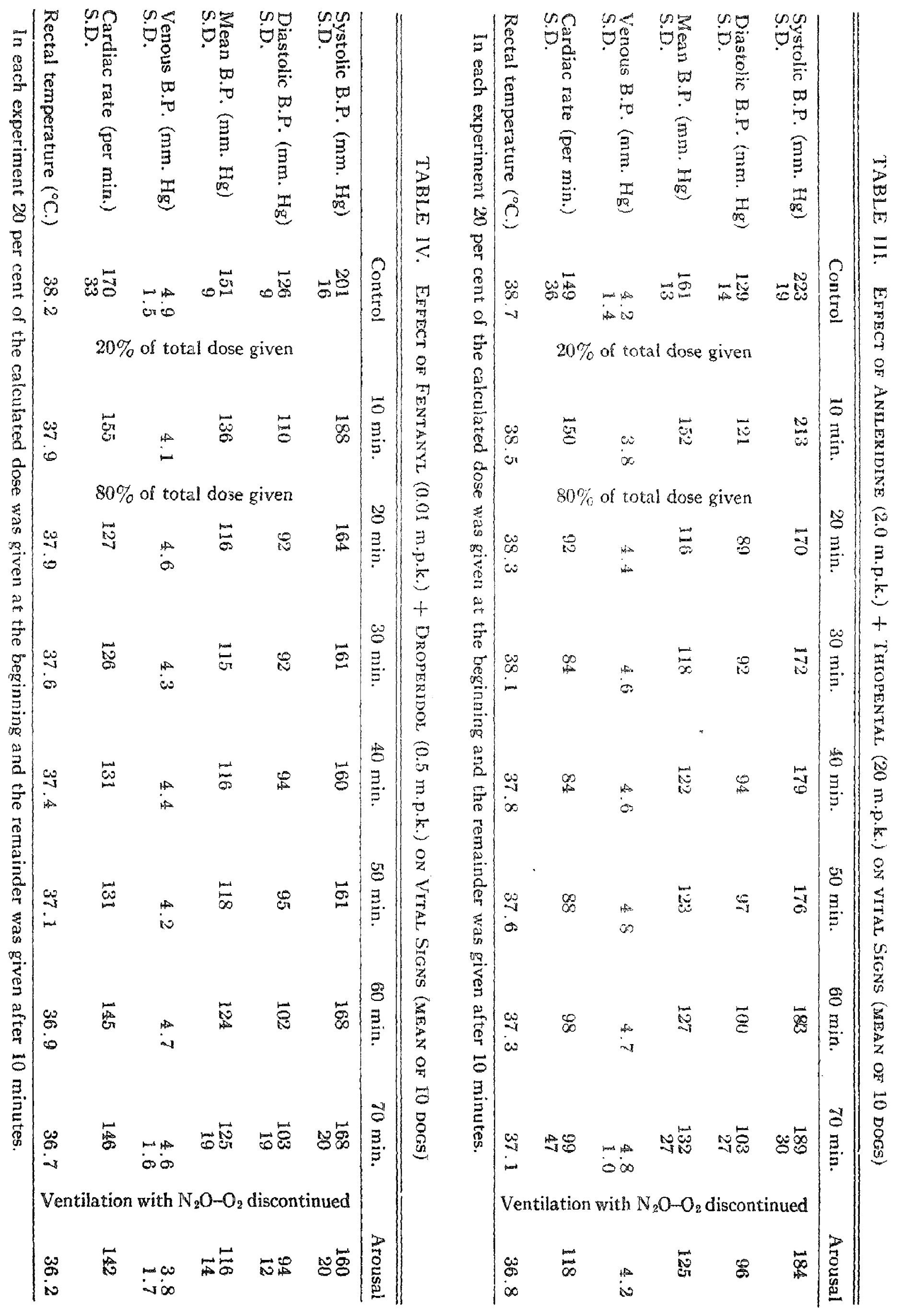




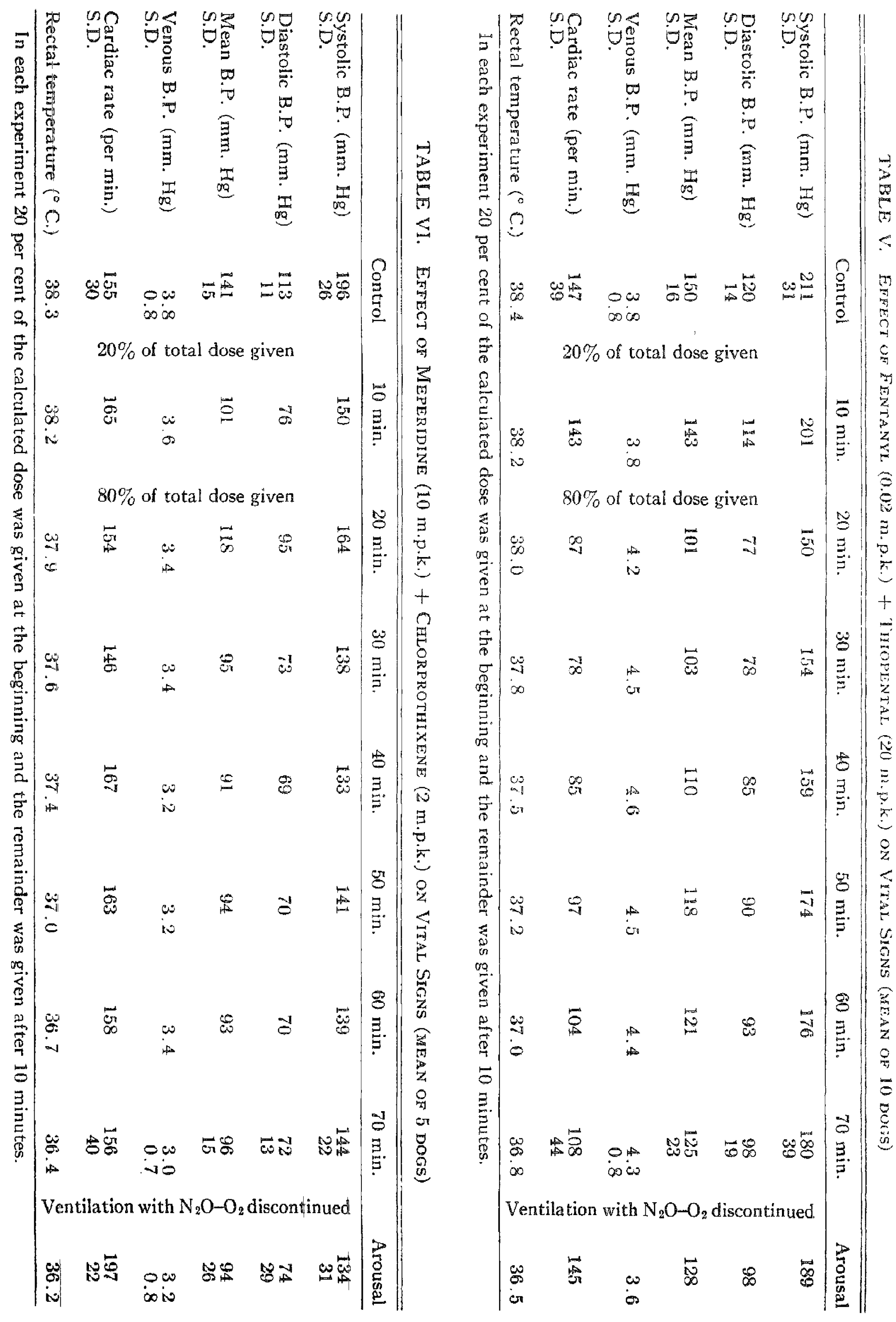




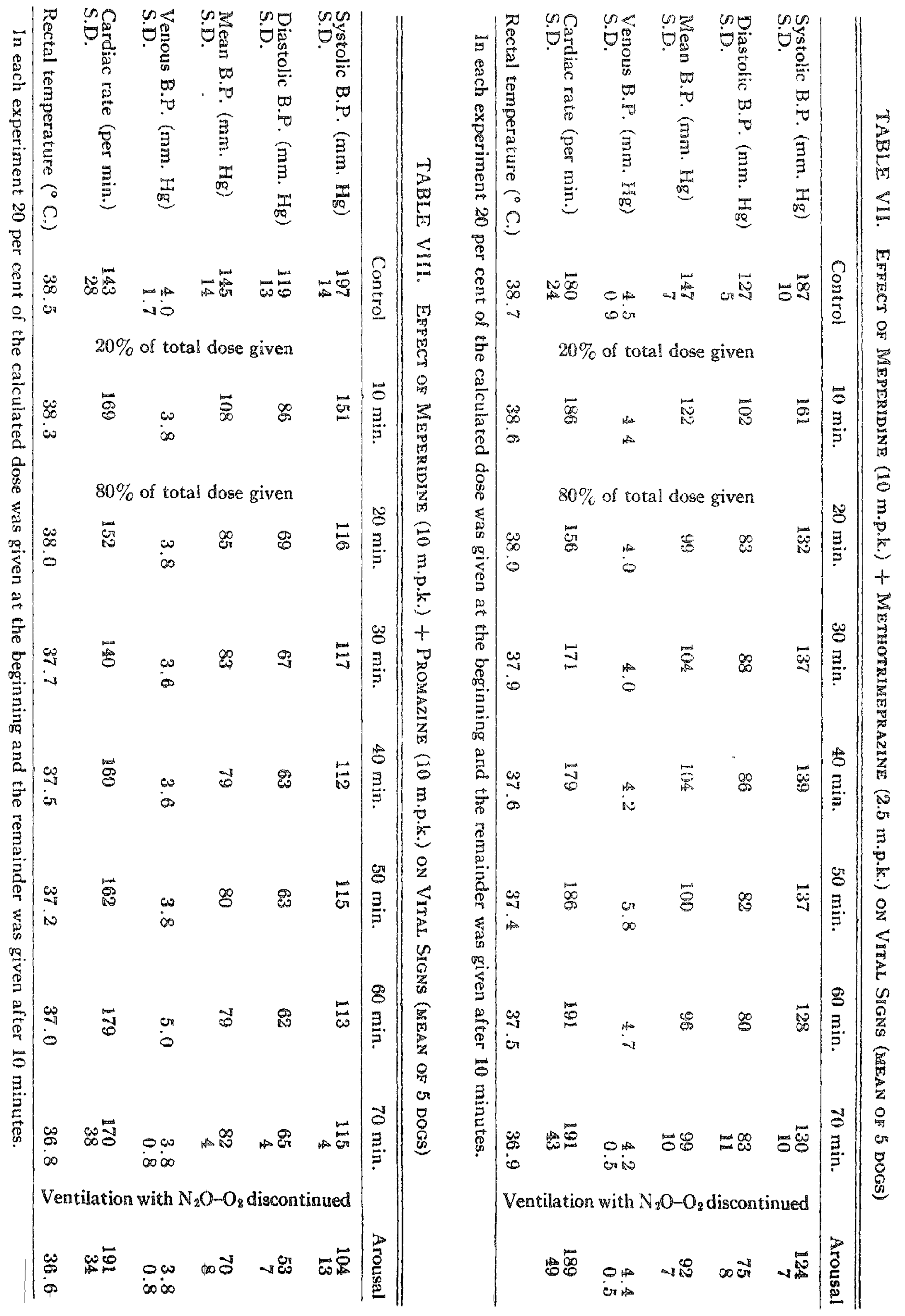




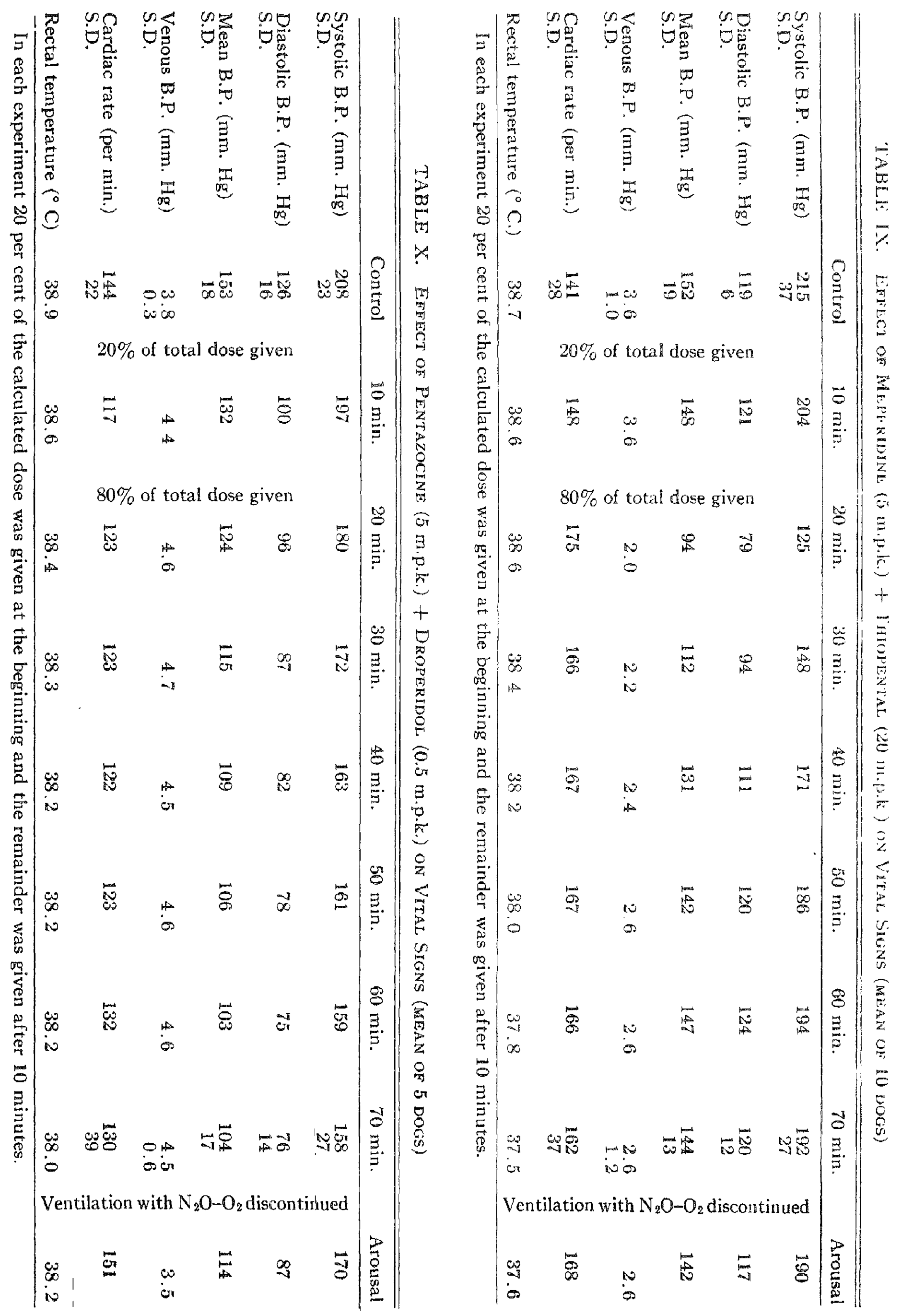




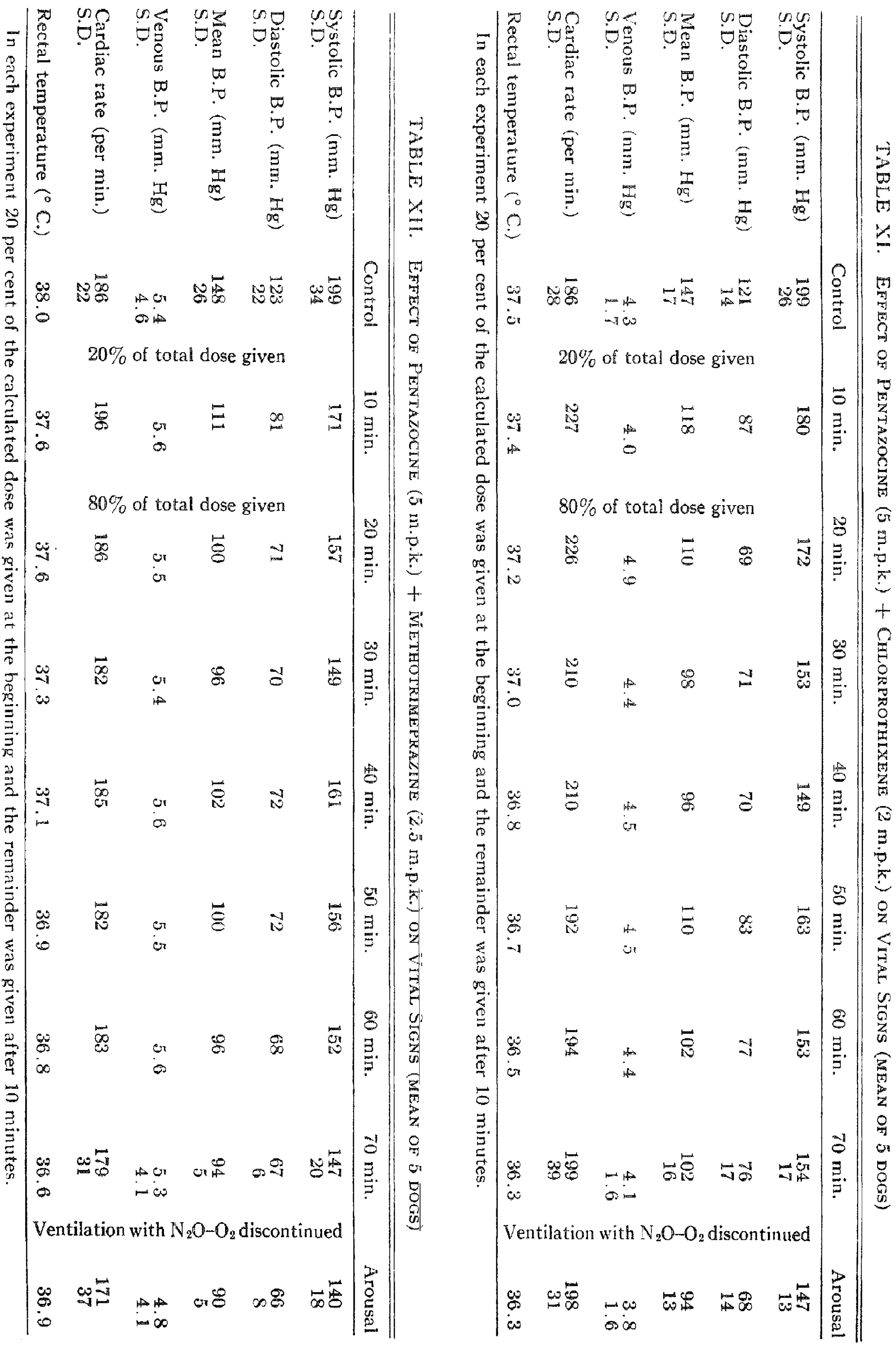




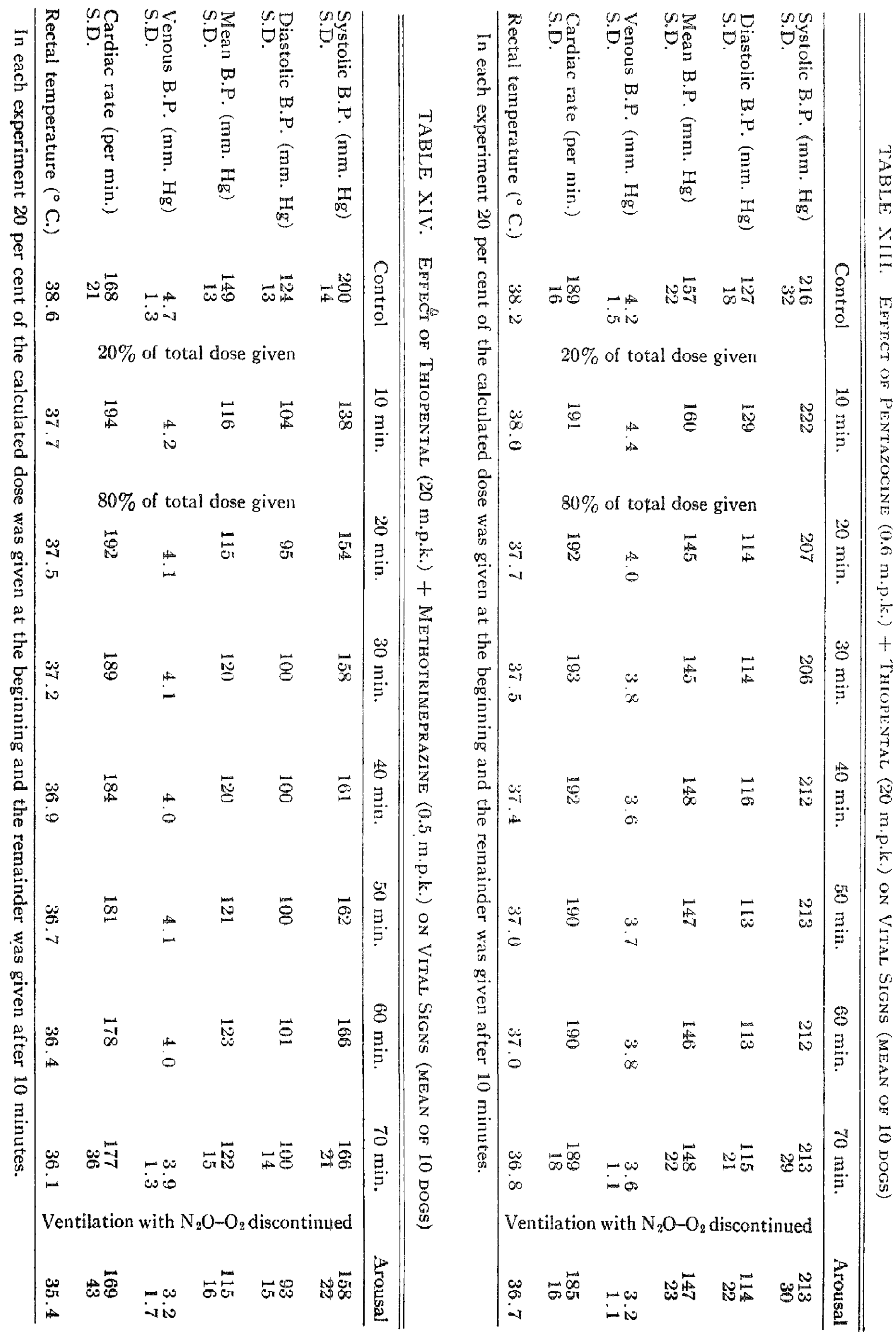




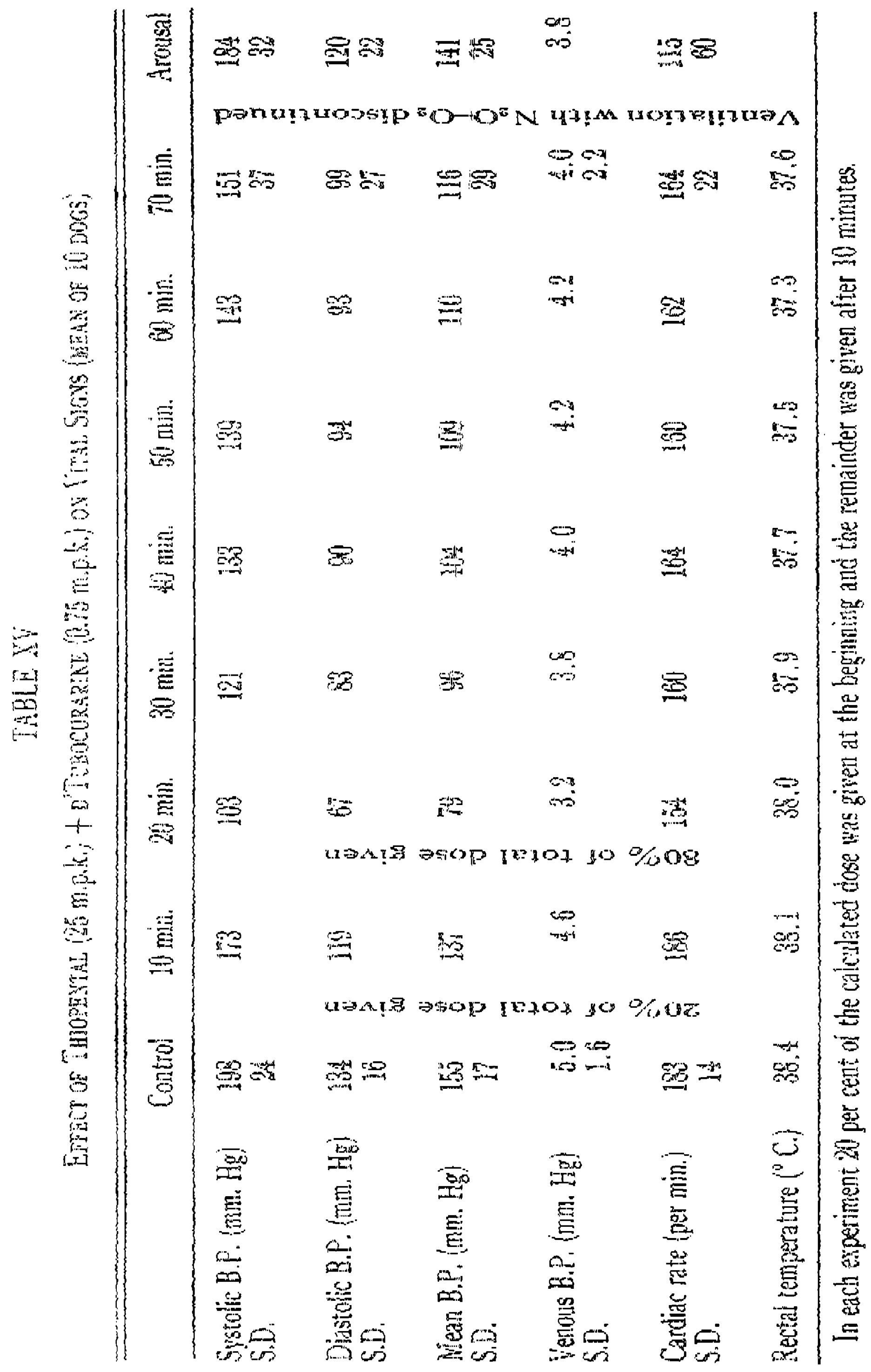


TABLE XVI

Effect of Anileridine (2.5 M.P.K.) + Chlorprothixene (2 M.P.K.) on Metabolism (MEAN OF 5 DOGS)

\begin{tabular}{lccc}
\hline \hline & Control & 70 min. & Arousal \\
\hline pH at $37.5^{\circ} \mathrm{C}$. & 7.43 & 7.35 & 7.23 \\
S.D. & 0.08 & 0.09 & 0.05 \\
Arterial $\mathrm{PCO}_{2}$ (mm. Hg) & 25 & 27 & 43 \\
S.D. & 4 & 6 & 4 \\
Plasma $\mathrm{HCO}_{3}^{-} /(\mathrm{mM}$./L.) & 15.2 & 13.7 & 16.6 \\
S.D. & 0.5 & 0.8 & 1.6 \\
Arterial $\mathrm{pO}_{2}$ (mm. Hg) & 152 & 153 & 328 \\
S.D. & 26 & 30 & 63 \\
Venous haematocrit & 46 & 37 & 35 \\
S.D. & 3 & 6 & 5 \\
Serum potassium (mEq./L.) & 4.9 & 4.0 & \\
S.D. & 0.4 & 0.6 & \\
Blood sugar (mg./100 ml.) & 127 & 220 & \\
S.D. & 16 & 42 & \\
Blood urea nitrogen (mg./100 ml.) & 12.2 & 11.8 & \\
S.D. & 2.9 & 2.8 & \\
SGO.T units & 40 & 37 & \\
S.D. & 5.2 & 6.2 & \\
SGP.T units & 27 & 29 & 3.5 \\
S.D. & 7.0 & \\
\hline
\end{tabular}

TABLE XVII

EFfect of ANileridine (2.5 M.P.K.) + Methotrimeprazine (2 M.P.K.) on Metabolism (MEAN OF 5 DOGS)

\begin{tabular}{lccc}
\hline & Control & 70 min. & Arousal \\
\hline pH at $37.5^{\circ} \mathrm{C}$. & 7.37 & 7.38 & 7.27 \\
S.D. & 0.07 & 0.09 & 0.05 \\
Arterial $\mathrm{COO}_{2}$ (mın. Hg) & 28 & 28 & 42 \\
S.D. & 7 & 7 & 2 \\
Plasma $\mathrm{HCO}_{3}-/(\mathrm{mM} . / \mathrm{L}$. .) & 15.3 & 15.3 & 18.3 \\
S.D. & 0.7 & 0.9 & 2.0 \\
Arterial $\mathrm{pO}_{2}$ (mm. Hg) & 143 & 137 & 298 \\
S.D. & 24 & 25 & 73 \\
Venous haematocrit & 12 & 36 & 37 \\
S.D. & 10 & 6 & 4 \\
Serum potassium (mEq./L.) & 4.9 & 4.1 & \\
S.D. & 0.3 & 0.8 & \\
Blood sugar (mg./100 ml.) & 108 & 138 & \\
S.D. & 16 & 43 & \\
Blood urea nitrogen (mg./100 ml.) & 10.6 & 10.0 & \\
S.D. & 0.5 & 0.7 & \\
SGO-T units & 38 & 37 & \\
S.D. & 10.2 & 3.7 & \\
SGP-T units & 30 & 29 & \\
S.D. & 12.5 & 14.2 & \\
\hline
\end{tabular}


TABLE XVIII

Effect of Anileridine (2.0 M.P.K.) + "Thiopental (20 M.P.K.) on Metabolism (MEAN OF 10 DOGS)

\begin{tabular}{lccc}
\hline \hline & Control & $70 \mathrm{~min}$. & Arousal \\
\hline pH at $37.5^{\circ} \mathrm{C}$. & 7.42 & 7.45 & 7.28 \\
S.D. & 0.05 & 0.07 & 0.05 \\
Arterial $p \mathrm{CO}_{2}$ (mm. Hg) & 26 & 24 & 42 \\
S.D. & 4 & 4 & 5 \\
Plasma $\mathrm{HCO}_{3}^{-}$(mM./L.) & 16.0 & 15.7 & 18.1 \\
S.D. & 2.2 & 2.0 & 2.7 \\
Arterial $p \mathrm{O}_{2}$ (mm. Hg) & 187 & 150 & 347 \\
S.D. & 108 & 18 & 69 \\
Venous haematocrit & 55 & 43 & 45 \\
S.D. & 5 & 7 & 8 \\
Serum potassium (mEq./L.) & 5.1 & 4.2 & \\
S.D. & 0.4 & 0.3 & \\
Blood sugar (mg./100 ml.) & 121 & 133 & \\
S.D. & 20 & 19 & \\
Blood urea nitrogen (mg./100 ml.) & 11.5 & 11.5 & \\
S.D. & 2.4 & 2.3 & \\
SGO-T units & 44 & 42 & \\
S.D. & 7.3 & 7.0 & \\
SGP-T units & 40 & 42 & \\
S.D. & 7.8 & 8.2 & \\
\hline
\end{tabular}

TABLE XIX

Effect of Fentanyl (0.01 M.P.K.) + Droperidol (0.5 M.P.k.) on Metabolism (MEAN OF 10 DOGS)

\begin{tabular}{lccc}
\hline & Control & 70 min. & Arousal \\
\hline pH at $37.5^{\circ} \mathrm{C}$. & 7.40 & 7.39 & 7.27 \\
S.D. & 0.05 & 0.05 & 0.05 \\
Arterial $p \mathrm{CO}_{2}$ (mm. Hg) & 25 & 22 & 33 \\
S.D. & 6 & 5 & 7 \\
Plasma $\mathrm{HCO}_{3}-$ (mM./L.) & 14.3 & 12.2 & 14.2 \\
S.D. & 2.6 & 2.2 & 2.8 \\
Arterial $p \mathrm{O}_{2}$ (mm. Hg) & 136 & 139 & 267 \\
S.D. & 21 & 14 & 96 \\
Venous haematocrit & 40 & 38 & 37 \\
S.D. & 8 & 4 & 4 \\
Serum potassium (mEq./L.) & 4.5 & 3.6 & \\
S.D. & 0.4 & 0.4 & \\
Blood sugar (mg./100 ml.) & 138 & 187 & \\
S.D. & 22 & 41 & \\
Blood urea nitrogen (mg./100 ml.) & 11.4 & 10.7 & \\
S.D. & 2.5 & 2.4 & \\
SGO-T units & 39 & 44 & \\
S.D. & 6.6 & 6.7 & \\
SGP-T units & 37 & 43 & \\
S.D. & 8.7 & 10.4 & \\
\hline
\end{tabular}


TABLE XX

EfFect of Fentanyl (0.02 M.P.K.) + Thiopental (20 M.P.K.) on Metaiolism (MEAN OF 10 DOGS)

\begin{tabular}{lccc}
\hline & Control & 70 min. & Arousal \\
\hline pH at $37.5^{\circ} \mathrm{C}$. & 7.47 & 7.48 & 7.30 \\
S.D. & 0.06 & 0.07 & 0.07 \\
Arterial $p \mathrm{CO}_{2}$ (mm. Hg) & 23 & 22 & 40 \\
S.D. & 1 & 3 & 8 \\
Plasma $\mathrm{HCO}_{3}^{-}$(mM./L.) & 15.4 & 15.0 & 18.3 \\
S.D. & 1.9 & 1.4 & 2.5 \\
Arterial $p \mathrm{O}_{2}$ (mm. Hg) & 184 & 201 & 315 \\
S.D. & 121 & 146 & 72 \\
Venous haematocrit & 53 & 45 & 50 \\
S.D. & 8 & 5 & 5 \\
Serum potassium (mEq./L.) & 5.1 & 4.1 & \\
S.D. & 0.4 & 0.4 & \\
Blood sugar (mg./100 ml.) & 112 & 125 & \\
S.D. & 18 & 18 & \\
Blood urea nitrogen (mg./100 ml.) & 12.0 & 11.1 & \\
S.D. & 2.7 & 2.3 & \\
SGO-T units & 46 & 45 & \\
S.D. & 18.1 & 19.1 & \\
SGP-T units & 41 & 42 & \\
S.D. & 21.7 & 21.6 & \\
\hline
\end{tabular}

TABLE XXI

Effect of Meperidine (10 M.P.K.) + Chlorprothixene (2 m.ip.K.) on Metabolism (MEAN OF 5 DOGS)

\begin{tabular}{|c|c|c|c|}
\hline & Control & $70 \mathrm{~min}$. & Arousal \\
\hline $\begin{array}{l}\text { pH at } 37.5^{\circ} \mathrm{C} \text {. } \\
\text { S.D. }\end{array}$ & $\begin{array}{l}7.39 \\
0 \\
09\end{array}$ & $\begin{array}{l}7.33 \\
0.09\end{array}$ & $\begin{array}{l}7.22 \\
0.03\end{array}$ \\
\hline $\begin{array}{l}\text { Arterial } p \mathrm{CO}_{2}(\mathrm{~mm} . \mathrm{Hg}) \\
\text { S.D. }\end{array}$ & $\begin{array}{r}28 \\
7\end{array}$ & $\begin{array}{r}29 \\
9\end{array}$ & $\begin{array}{r}41 \\
3\end{array}$ \\
\hline $\begin{array}{l}\text { Plasma } \mathrm{HCO}_{3}^{-}(\mathrm{mM} . / \mathrm{L} .) \\
\text { S.D. }\end{array}$ & $\begin{array}{rl}15 & 7 \\
0 & 9\end{array}$ & $\begin{array}{r}13.7 \\
0.9\end{array}$ & $\begin{array}{r}15.6 \\
0.7\end{array}$ \\
\hline $\begin{array}{l}\text { Arterial } p \mathrm{O}_{2}(\mathrm{~mm} . \mathrm{Hg}) \\
\text { S.D. }\end{array}$ & $\begin{array}{r}166 \\
22\end{array}$ & $\begin{array}{r}175 \\
25\end{array}$ & $\begin{array}{r}296 \\
75\end{array}$ \\
\hline $\begin{array}{l}\text { Venous haematocrit } \\
\text { S.D. }\end{array}$ & $\begin{array}{r}46 \\
4\end{array}$ & $\begin{array}{r}38 \\
4\end{array}$ & $\begin{array}{r}37 \\
5\end{array}$ \\
\hline $\begin{array}{l}\text { Serum potassium (mEq./L.) } \\
\text { S.D. }\end{array}$ & $\begin{array}{l}4.7 \\
0.2\end{array}$ & $\begin{array}{l}3.4 \\
0.1\end{array}$ & \\
\hline $\begin{array}{l}\text { Blood sugar (mg. } / 100 \mathrm{ml} \text {.) } \\
\text { S.D. }\end{array}$ & $\begin{array}{r}117 \\
11\end{array}$ & $\begin{array}{r}150 \\
16\end{array}$ & \\
\hline $\begin{array}{l}\text { Blood urea nitrogen }(\mathrm{mg} . / 100 \mathrm{ml} \text {.) } \\
\text { S.D. }\end{array}$ & $\begin{array}{rr}11 & 0 \\
2 & 3\end{array}$ & $\begin{aligned} 10.4 \\
2.1\end{aligned}$ & \\
\hline $\begin{array}{l}\text { SGO-T units } \\
\text { S.D. }\end{array}$ & $\begin{array}{l}37 \\
8.5\end{array}$ & $\begin{array}{r}37 \\
6.5\end{array}$ & \\
\hline $\begin{array}{l}\text { SGP-T units } \\
\text { S.D. }\end{array}$ & $\begin{array}{l}31 \\
3.9\end{array}$ & $\begin{array}{l}34 \\
3.6\end{array}$ & \\
\hline
\end{tabular}


TABLE XXII

Effect of Meperidine (10 M.P.K.) + Methot Rimeprazine (2.5 M.P.K.) on Metabolism (MEAN OF 5 DOGS)

\begin{tabular}{lccc}
\hline \hline & Control & 70 min. & Arousal \\
\hline pH at $37.5^{\circ} \mathrm{C}$. & 7.37 & 7.32 & 7.30 \\
S.D. & 0.04 & 0.09 & 0.06 \\
Arterial $p \mathrm{CO}_{2}$ (mm. Hg) & 28 & 30 & 32 \\
S.D. & 5 & 5 & 5 \\
Plasma $\mathrm{HCO}_{3}-$ (mM./L.) & 15.3 & 14.6 & 15.3 \\
S.D. & 26 & 3.0 & 3.3 \\
Arterial $p \mathrm{O}_{2}$ (mm. Hg) & 149 & 145 & 239 \\
S.D. & 22 & 36 & 87 \\
Venous haematocrit & 37 & 35 & 34 \\
S.D. & 11 & 6 & 6 \\
Serum potassium (mEq./L.) & 4.8 & 3.5 & \\
S.D. & 0.6 & 0.6 & \\
Blood sugar (mg./100 ml.) & 137 & 201 & \\
S.D. & 24 & 24 & \\
Blood urea nitrogen (mg./100 ml.) & 12.5 & 11.5 & \\
S.D. & 1.9 & 1.9 & \\
SGO-T units & 36 & 34 & \\
S.D. & 50 & 5.3 & \\
SGP.T units & 27 & 33 & 4.7 \\
S.D. & & & \\
\hline
\end{tabular}

TABLE XXIII

Effect of Meperidine (10 m.P.K.) + Phomazine (10 M.P.K.) on Metabolism (MEAN OF DOGS)

\begin{tabular}{lccc}
\hline & Control & 70 min. & Arousal \\
\hline pH at $37.5^{\circ} \mathrm{C}$. & 7.41 & 7.34 & 7.24 \\
S.D. & 0.08 & 0.04 & 0.04 \\
Arterial $p \mathrm{CO}_{2}$ (mm. Hg) & 26 & 28 & 39 \\
S.D. & 1 & 4 & 4 \\
Plasma $\mathrm{HCO}_{3}^{-}$(mM./L.) & 15.9 & 14.0 & 15.5 \\
S.D. & 15 & 2.0 & 17 \\
Arterial $p \mathrm{O}_{2}$ (mm. Hg) & 152 & 162 & 326 \\
S.D. & 16 & 31 & 55 \\
Venous haematocrit & 48 & 40 & 39 \\
S.D. & 7 & 5 & 6 \\
Serum potassium (mEq./L.) & 4.8 & 3.6 & \\
S.D. & 0.3 & 0.2 & \\
Blood sugar (mg./100 ml.) & 112 & 154 & \\
S.D. & 17 & 33 & \\
Blood urea nitrogen (mg./100 ml.) & 10 & 11 & \\
S.D. & 1.8 & 2.8 & \\
SGO-T units & 40 & 44 & \\
S.D. & 15.8 & 14.7 & \\
SGP.T units & 33 & 39 & \\
S.D. & 18.2 & 18.8 \\
\hline
\end{tabular}


TABLE XXIV

Effect of Meperidine (5 M.P.K.) + Thiopental (20 M.P.K.) on Metabolism (MEAN OF 10 DOGS)

\begin{tabular}{lccc}
\hline & Control & 70 min. & Arousal \\
\hline pH at $37.5^{\circ} \mathrm{C}$. & 7.46 & 7.39 & 7.30 \\
S.D. & 0.09 & 0.10 & 0.07 \\
Arterial $p \mathrm{CO}_{2}$ (mm. Hg) & 26 & 30 & 41 \\
S.D. & 6 & 6 & 7 \\
Plasma $\mathrm{HCO}_{3}^{-}$(mM./L.) & 17.0 & 17.0 & 18.6 \\
S.D. & 3.6 & 3.8 & 4.3 \\
Arterial $\mathrm{O}_{2}$ (mm. Hg) & 152 & 134 & 228 \\
S.D. & 18 & 30 & 122 \\
Venous haematocrit & 53 & 53 & 54 \\
S.D. & 6 & 7 & 6 \\
Serum potassium (mEq./L.) & 5.1 & 4.3 & \\
S.D. & 0.3 & 0.6 & \\
Blood sugar (mg./100 ml.) & 108 & 123 & \\
S.D. & 21 & 18 & \\
Blood urea nitrogen (mg./100 ml.) & 11.2 & 11.7 & \\
S.D. & 2.3 & 2.4 & \\
SGO-T units & 40 & 40 & \\
S.D. & 12.6 & 12.1 & \\
SGP-T units & 35 & 38 & \\
S.D. & 8.7 & 8.3 & \\
\hline
\end{tabular}

TABLE XXV

Effect of Pentazocine (5 m.P.K.) + Droperidol (0.5 M.P.K.) on Metabolism (MEAN OF 5 DOGS)

\begin{tabular}{lccc}
\hline & Control & 70 min. & Arousal \\
\hline pH at $37.5^{\circ} \mathrm{C}$. & 7.49 & 7.33 & 7.33 \\
S.D. & 0.07 & 0.11 & 0.06 \\
Arterial $\mathrm{CO}_{2}$ (mm. Hg) & 22 & 27 & 26 \\
S.D. & 6 & 10 & 5 \\
Plasma $\mathrm{HCO}_{3}-(\mathrm{mM} . / \mathrm{L})$. & 15.2 & 13.0 & 13.0 \\
S.D. & 3.1 & 2.6 & 2.4 \\
Arterial $\mathrm{pO}_{2}$ (mm. Hg) & 164 & 172 & 299 \\
S.D. & 10 & 15 & 76 \\
Venous haematocrit & 52 & 48 & 45 \\
S.D. & 5 & 4 & 4 \\
Serum potassium (mEq./L.) & 5.8 & 3.5 & \\
S.D. & 0.4 & 0.5 & \\
Blood sugar (mg./100 ml.) & 114 & 176 & \\
S.D. & 18 & 79 & \\
Blood urea nitrogen (mg./100 ml.) & 11.4 & 10.8 & \\
S.D. & 2.0 & 2.0 & \\
SGO-T units & 49 & 57 & \\
S.D. & 6.0 & 11.3 & \\
SGP-T units & 39 & 50 & \\
S.D. & 6.0 & 7.1 & \\
\hline
\end{tabular}


TABLE XXVI

Effect of pentazocine (5 M.P.K.) + Chlorprothixene (2 M.P.K.) on Metabolism (MEAN OF 5 DOGS)

\begin{tabular}{lccc}
\hline \hline & Control & $70 \mathrm{~min}$. & Arousal \\
\hline pH at $37.5^{\circ} \mathrm{C}$. & 7.41 & 7.27 & 7.21 \\
S.D. & 0.04 & 0.05 & 0.06 \\
Arterial $\mathrm{pCO}_{2}$ (mm. Hg) & 23 & 34 & 39 \\
S.D. & 4 & 5 & 8 \\
Plasma $\mathrm{HCO}_{3} \quad$ (mM./L.) & 14.8 & 9.9 & 11.0 \\
S.D. & 4.8 & 3.1 & 3.8 \\
Arterial $\mathrm{pO}_{2}$ (mm. Hg) & 191 & 171 & 177 \\
S.D. & 70 & 19 & 35 \\
Venous haematocrit & 52 & 38 & 37 \\
S.D. & 7 & 5 & 5 \\
Serum potassium (mEq./L.) & 4.5 & 3.3 & \\
S.D. & 0.4 & 0.8 & \\
Blood sugar (mg./100 ml.) & 102 & 234 & \\
S.D. & 27 & 40 & \\
Blood urea nitrogen (mg./100 ml.) & 14.8 & 14.6 & \\
S.D. & 4.9 & 4.8 & \\
SGO-T units & 40 & 42 & \\
S.D. & 10.3 & 6.5 & \\
SGP.T units & 36 & 40 & \\
S.D. & 10.4 & 5.9 & \\
\hline
\end{tabular}

TABLE XXVII

Effect of Pentazocine (5 M.P.K.) + Methotrimeprazine (2.5 M.P.K.) on Metabolism (MEAN OF 5 DOGS)

\begin{tabular}{lccc}
\hline & Control & $70 \mathrm{~min}$. & Arousal \\
\hline pH at $37.5^{\circ} \mathrm{C}$. & 7.42 & 7.31 & 7.19 \\
S.D. & 0.05 & 0.02 & 0.04 \\
Arterial $\mathrm{pCO}_{2}$ (mm. Hg) & 21 & 22 & 33 \\
S.D. & 4 & 4 & 7 \\
Plasma $\mathrm{HCO}_{3}-(\mathrm{mM} . / \mathrm{L})$. & 12.6 & 10.4 & 12.0 \\
S.D. & 1.4 & 1.6 & 1.9 \\
Arterial $\mathrm{pO}_{2}$ (mm. Hg) & 155 & 169 & 274 \\
S.D. & 23 & 26 & 53 \\
Venous haematocrit & 47 & 37 & 40 \\
S.D. & 8 & 4 & 6 \\
Serum potassium (mEq./L.) & 4.6 & 2.7 & \\
S.D. & 0.1 & 0.3 & \\
Blood sugar (mg./100 ml.) & 112 & 230 & \\
S.D. & 10 & 25 & \\
Blood urea nitrogen (mg./100 ml.) & 14.0 & 14.4 & \\
S.D. & 2.7 & 2.6 & \\
SGO-T units & 16 & 34 & \\
S.D. & 15.1 & 13.5 & \\
SGP.T units & 33 & 41 & \\
S.D. & 21.8 & 11.7 & \\
\hline
\end{tabular}


TABLE XXVIII

Effect of Pentazocine (0.6 M.P.K.) + Thiopental (20 M.P.K.) on Metabolism (MEAN OF 10 DOGS)

\begin{tabular}{lccc}
\hline & Control & 70 min. & Arousal \\
\hline pH at $37.5^{\circ} \mathrm{C}$. & 7.38 & 7.34 & 7.23 \\
S.D. & 0.06 & 0.06 & 0.05 \\
Arterial $p \mathrm{CO}_{2}$ (mm. Hg) & 23 & 23 & 36 \\
S.D. & 4 & 5 & 8 \\
Plasma $\mathrm{HCO}_{3}^{-}(\mathrm{mM} . / \mathrm{L})$. & 12.6 & 11.8 & 13.9 \\
S.D. & 11 & 1.5 & 1.7 \\
A.rterial $p \mathrm{O}_{2}$ (mm. Hg) & 152 & 164 & 217 \\
S.D. & 27 & 33 & 77 \\
Venous haematocrit & 51 & 48 & 50 \\
S.D. & 4 & 5 & 5 \\
Serum potassium (mEq./L.) & 4.4 & 3.0 & \\
S.D. & 0.5 & 0.5 & \\
Blood sugar (mg./100 ml.) & 121 & 150 & \\
S.D. & 27 & 36 & \\
Blood urea nitrogen (mg./100 ml.) & 15.1 & 14.1 & \\
S.D. & 4.2 & 3.6 & \\
SGO-T units & 41 & 47 & \\
S.D. & 14.9 & 15.4 & \\
SGP.T units & 52 & 54 & \\
S.D. & 22.1 & 21.8 & \\
\hline
\end{tabular}

TABLE XXIX

EFFect of Thiopental (20 M.P.K.) + Methotrimeprazine (0.5 M.P.K.) on Metabolism (MEAN OF 10 DOGS)

\begin{tabular}{lccc}
\hline & Control & 70 min. & Arousal \\
\hline pH at $37.5^{\circ} \mathrm{C}$. & 7.40 & 7.41 & 7.25 \\
S.D. & 0.04 & 0.07 & 0.09 \\
Arterial $p \mathrm{CO}_{2}$ (mm. Hg) & 25 & 25 & 43 \\
S.D. & 5 & 6 & 13 \\
Plasma $\mathrm{HCO}_{3}-(\mathrm{mM} . / \mathrm{L})$. & 14.7 & 14.3 & 17.2 \\
S.D. & 19 & 2.2 & 2.1 \\
Arterial $p \mathrm{O}_{2}$ (mm. Hg) & 125 & 143 & 290 \\
S.D. & 30 & 11 & 21 \\
Venous haematocrit & 44 & 36 & 36 \\
S.D. & 5 & 5 & 4 \\
Serum potassium (mEq./L.) & 4.2 & 3.2 & \\
S.D. & 0.5 & 0.6 & \\
Blood sugar (mg./100 ml.) & 140 & 176 & \\
S.D. & 22 & 30 & \\
Blood urea nitrogen (mg./100 ml.) & 12.3 & 12.2 & \\
S.D. & 3.1 & 3.1 & \\
SGO-T units & 41 & 39 & \\
S.D. & 9.4 & 16.2 & \\
SGP.T units & 40 & 40 & \\
S.D. & 18.4 & 17.3 & \\
S. & & &
\end{tabular}


TABLE XXX

Effect of Thiopental (25 M.P.K.) + $d$ 'Tubocurarine (0.75 M.P.K.) on MEtabolism (MEAN OF 10 DOGS)

\begin{tabular}{lccc}
\hline \hline & Control & $70 \mathrm{~min}$ & Arousal \\
\hline pH at $37.5^{\circ} \mathrm{C}$. & 7.30 & 7.30 & 6.96 \\
S.D. & $\mathbf{0 . 0 5}$ & $\mathbf{0 . 1 0}$ & $\mathbf{0 . 2 3}$ \\
Arterial $p \mathrm{CO}_{2}(\mathrm{~mm} . \mathrm{Hg})$ & 33 & 30 & 104 \\
S.D. & 8 & 10 & 54 \\
Plasma $\mathrm{HCO}_{3}^{-}(\mathrm{mM} . / \mathrm{L})$. & 14.7 & 13.3 & \\
S.D. & 2.0 & 3.3 & \\
Arterial $\mathrm{pO}_{2}$ (mm. Hg) & 137 & 143 & 179 \\
S.D. & 19 & 21 & 91 \\
Venous haematocrit & 416 & 45 & 50 \\
S.D. & 7 & 10 & 14 \\
Serum potassium (mEq./L.) & 4.5 & 3.8 & \\
S.D. & 0.2 & 0.5 & \\
Blood sugar (mg./100 ml.) & 138 & 154 & \\
S.D. & $4: 3$ & 16 & \\
Blood urea nitrogen (mg./100 ml.) & 10.6 & 10.6 & \\
S.D. & 2.1 & 2.1 & \\
SGO-T units & 36 & 36 & \\
S.D. & 8.7 & 14.3 & \\
SGP.T units & 34 & 42 & \\
S.D. & 14.5 & 20.8 & \\
\hline
\end{tabular}

pentazocine, and d'tubocurarine). In all of these tests, induction of anaesthesia was smooth and the dogs remained relatively quiet throughout the anaesthetic period. A few animals blinked their eyes from time to time in response to noise and some moved when blood samples were drawn, but, for the most part, the anaesthetics were considered adequate.

Among the above mixtures, the least post-anaesthetic respiratory depression was observed with the fentanyl-droperidol mixture $\left(p \mathrm{CO}_{2}\right.$ rose $8 \mathrm{~mm}$. at the time of recovery) and the most depression was consistently observed when the thiopental-d'tubocurarine mixture (Baird's solution $\left.{ }^{2}\right)$ was used $\left(p \mathrm{CO}_{2}\right.$ rose $74 \mathrm{~mm}$.). The change was remarkably consistent with the other mixtures $\left(p \mathrm{CO}_{2}\right.$ rose 13 to $18 \mathrm{~mm}$.). No significant changes occurred in the blood pressure, and the heart rate usually decreased.

The mixtures containing the analgesics anileridine, meperidine, and pentazocine combined with the neuroleptics chlorprothixene, droperidol, methotrimeprazine, and promazine all proved to be unsatisfactory for one reason or another, even after considerable manipulation of the dosages in preliminary experiments. The most prominent undesirable feature was our awareness that the level of anaesthesia frequently became so light that from moment to moment we did not know whether we could hold back the administration of a supplementary dose of the mixture in order to maintain smooth anaesthesia, as evidenced by the dog blinking its eyes, gazing about, moving his limbs, chewing on the endotracheal tube, and raising the head in response to noise while, at the same time, the mean blood pressure was reduced at least to a level which we considered 


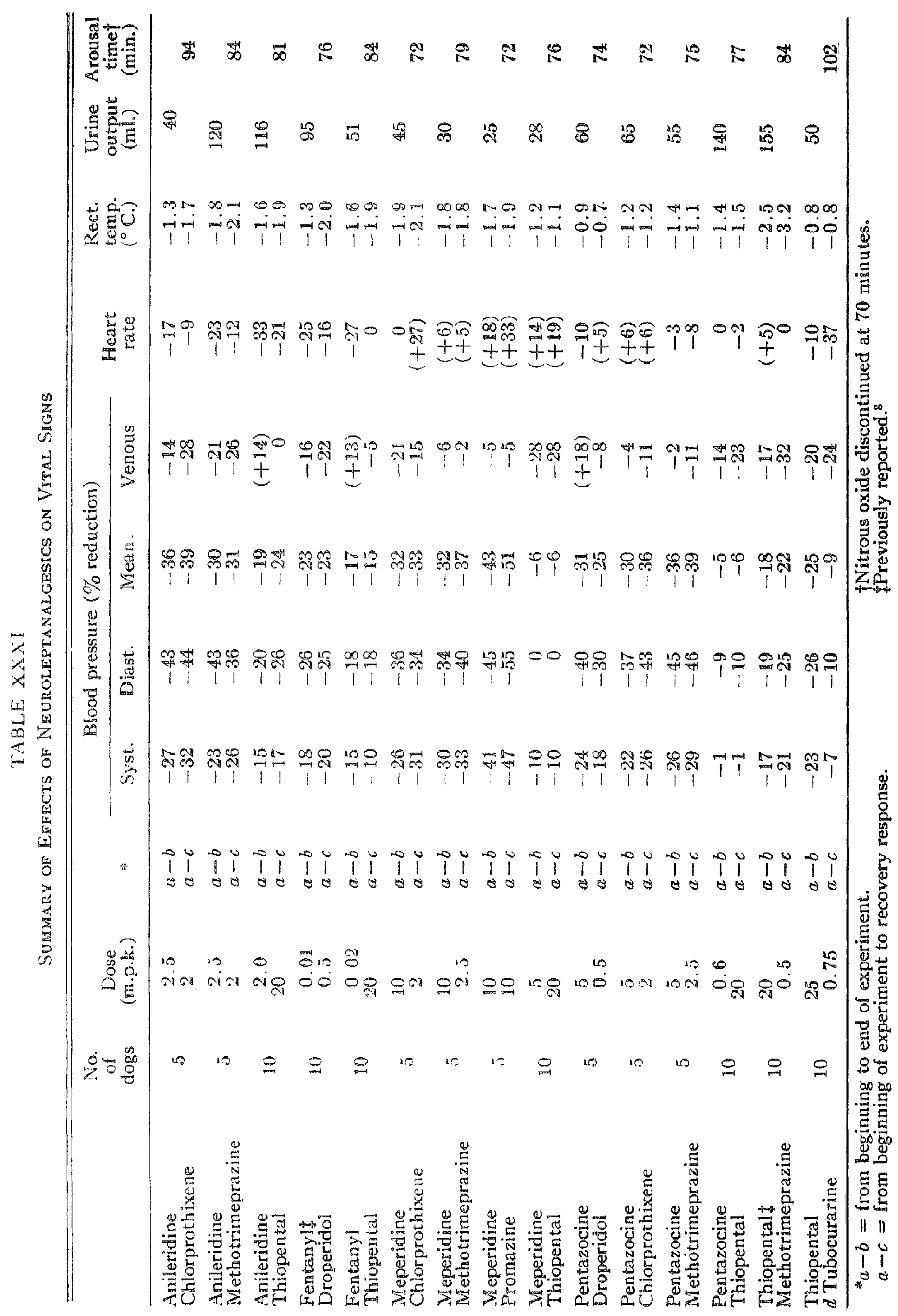




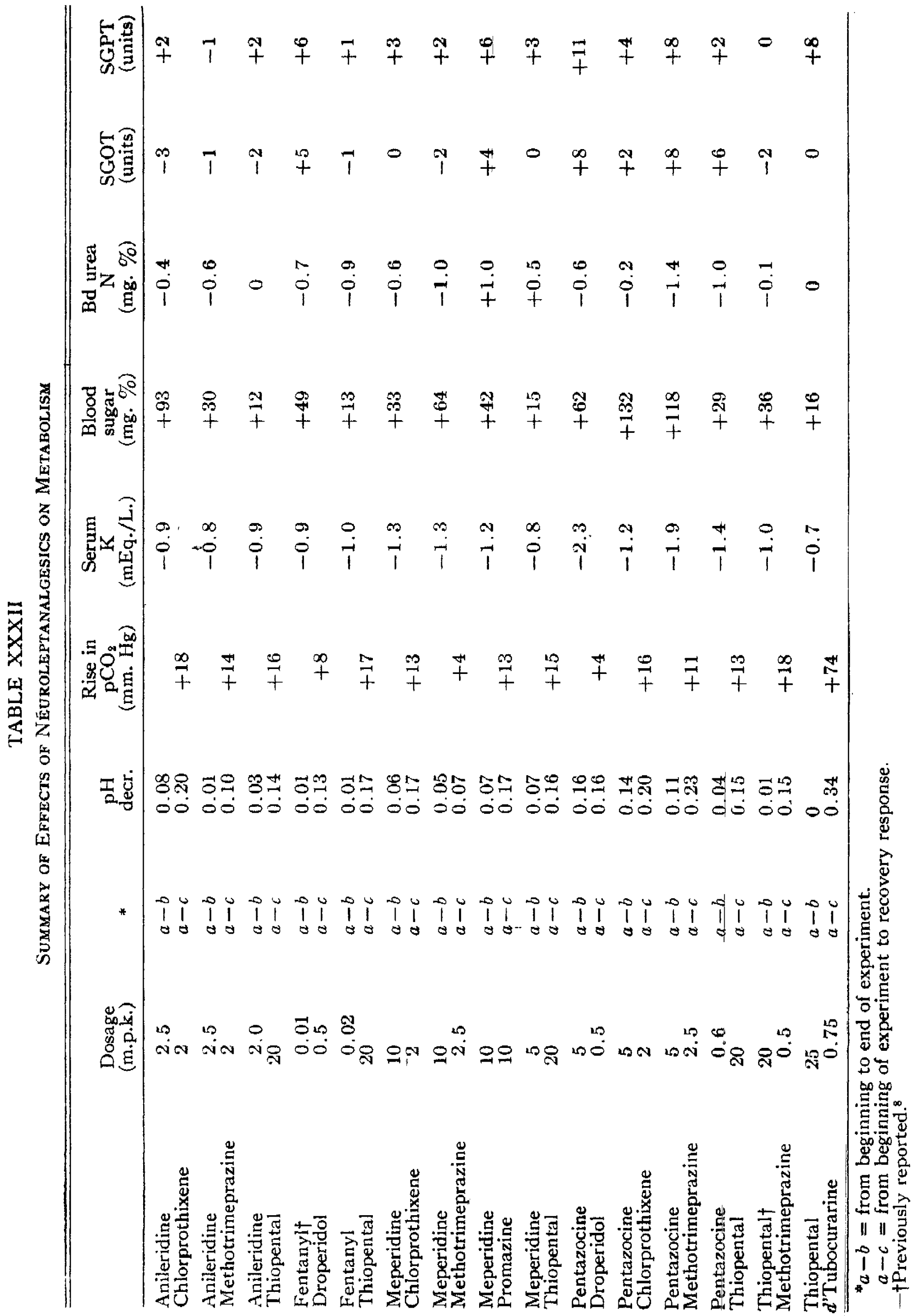


excessively below the control reading $(>30 \%)$. Very often additional drugs had to be given to keep the animals quiet.

In none of the experiments did the electrocardiogram (lead 2) show alterations that have any significance.

Rectal temperature was invariably decreased during the course of all of the experiments, usually in excess of $1^{\circ} \mathrm{C}$.

Defaecation occurred during a few experiments: one dog had a bloody stool during anaesthesia with anileridine-thiopental and one with meperidine-thiopental, and two dogs passed a stool during fentanyl-thiopental anaesthesia. Most of the dogs defaecated during recovery after termination of the tests. None of the dogs had diarrhoea.

None of the dogs had retching or vomiting during the recovery period. A brief red flush of the forepaw occurred when the mixture of meperidine-thiopental was injected ( 3 dogs).

During anaesthesia, urine output was satisfactory with all the mixtures except those containing meperidine, and the anileridine-chlorprothixene mixture $(<50$ ml. urine). All of the mixtures except those containing thiopental caused an appreciable rise in the blood sugar. This observation has been mentioned previously. ${ }^{8}$ Although there was a consistent reduction in the serum potassium during anaesthesia with all the mixtures tested, only with pentazocine-droperidol was the change appreciable and probably excessive. The explanation for this change remains obscure. ${ }^{8}$

In all of the experiments, the changes in blood urea nitrogen, SGOT, and SGPT were negligible and of no clinical significance.

All the animals were awake within 30 minutes after the nitrous oxide was discontinued except those that received thiopental-d'tubocurarine. However, they were invariably unable to ambulate for a variable period of time after they were awake, extending from 30 minutes to some hours after the tests, usually because of weakness of the hind limbs. Since the femoral vessels were canulated in each dog, we were undecided as to whether the difficulty in ambulation was caused by the anaesthetic alone. All of the animals appeared to be quite normal the following day, although most of them slept on and off for several hours after each test. There did not appear to be any consistent difference in the recovery responses that could be specifically attributed to any of the mixtures.

Tests were repeated at intervals of not less than one week on the individual dogs, and with the preliminary tests most dogs had 8 anaesthetics, but none of the animals seemed to develop an aversion to the tests that might be interpreted as attributable to an unhappy psychic or sensory experience following a previous experiment.

\section{DisCUSSION}

The evaluation of the efficacy of intravenous amaesthetic agents presents a problem in semantics which is even more complex than that related to the study of analgesics ${ }^{33}$ and inhalation agents. ${ }^{34}$ Quantitative and qualitative testing involves more than merely measuring the responses of a number of physiological and pharmacological parameters and then stating: "this is good, and that is not 
good." When, we combine two or more intravenous agents to accomplish a smooth anaesthetic state, we are certainly dealing with a far more complicated situation that at the present time can only be evaluated empirically on the basis of the occurrence of undesirable responses. ${ }^{1}$

In the concluding remarks of his Joseph Clover Lecture in 1954, T. C. Gray said:

The speed of pharmacological discovery and the sudden impact of new conceptions such as hypotension and hypothermia is apt indeed to create occasional bewilderment. There is a temptation to react by developing a nostalgia for the old days when we were able to produce so much-indeed, far too much-with only one agent. If we succumb to this temptation, we talk of polypharmacy and tend to sneer perhaps a little at the 'cocktail' mentality which is developing. I have attempted to draw attention to the desirable principle, now practicable, of differential disintegration of the nervous system controlled by the anaesthetist, as opposed to the indiscriminate uncontrolled and therefore undesirable disintegration of ten years ago. This approach calls for, in American terminology, a new 'conceptual system,' a new thinking, and I would answer those who, in dismay at the effort required, ask the question 'Whither anaesthesia?' by quoting the great St. Augustine, 'Time, sirs, doth not rest, nor rolls it idly round about these senses of ours, but it worketh strange changes in the mind.'35

I have quoted Professor Gray on this subject for his words fit well into the current dilemma, which he foresaw.

As new and more potent analgesics and neurosedatives (neuroleptics) have been developed, we have become accustomed to the basic "cocktail" concept of Laborit and Huguenard. ${ }^{36}$ This idea has certainly permeated the everyday practice of anaesthesia during the past decade, as most of us now use potent sedatives, muscle relaxants, analgesics, and hypnotics in combinations to accomplish satisfactory surgical anaesthesia, without a second thought, although we may attach a different name to it. ${ }^{37}$

There is a new question which should now be answered: Is it reasonable to combine these potent agents for intravenous administration into fixed mixtures? In clinical medicine, such a practice has not yet been officially condoned by the AMA Council of Drugs, for, as Isaac Starr has stated clearly, when two active drugs are needed, they should be given separately so that their dosages can be manipulated separately in accordance with the needs of the patient. ${ }^{38}$ Nevertheless, some skilled anaesthetists are accustomed to using drug mixtures; they have been using them for a great many years, and they may be in the best position to test the efficacy of new drugs in combinations. Whenever their use proves unworthy of a situation, the anaesthetist can easily revert to using the specific single drug that seems to be indicated. It is only when one becomes rigid in the use of mixtures that serious problems can and do arise.

In evaluating the above series of combinations, we have kept the disadvantages of using a fixed mixture foremost in our minds and have come to the conclusion that for initiating an anaesthetic it is not inconvenient to employ a predetermined combination of drugs of evident usefulness. Thereafter, the supplementary requirements can be evaluated and judged best during the progress of a case by administering the individual compounds singly to fit the needs of the moment.

From the data reported above, it appears to us, at present, that six of the 
combinations of a potent analgesic and a neuroleptic tested seem to be satisfactory for inducing clinical anaesthesia when used along with nitrous oxide. One is the fentanyl-droperidol (1:50) mixture. This mixture is the only one tested for which the term "neuroleptanalgesia" can be applied as coined by the French psychiatrist Jean Delay, ${ }^{39}$ and first put into clinical practice by DeCastro and Mundeleer and by Nilsson. ${ }^{40-42}$ They conceived neuroleptanalgesia as a state of central nervous system depression and sensory deprivation which is produced without the use of barbiturates or volatile anaesthetic agents. ${ }^{43}$ The state created is one of total indifference in a conscious person who remains responsive to auditory stimuli and can deny awarene|ss to painful stimuli. Since we feel, along with others, that there is no advantage to having the patient awake during a surgical operation, there is also no particular reason for eliminating the use of an ultra-short-acting barbiturate, such as thiopental. After all, this was one of the earliest of the drugs that have now come to be known as neurosedatives or neuroleptics, for, as long ago as 1936, Horsley employed thiopental for therapeutic psychotherapy (narco-analysis). ${ }^{44}$ Since thiopentall is also a potent hypnotic of relatively short action, and, in very low dosage, acts also as a neurosedative, it is not surprising that satisfactory anaesthetic conditions and a smooth recovery were provided when it was combined with potent analgesic drugs (anileridine, fentanyl, meperidine, methotrimeprazine, and pentazocine). For years now, it has been used successfully with d'tubocurare and/or nitrous oxide for a wide variety of surgical procedures. ${ }^{2,45-48}$

As the efficacy of each pair of agents is analysed, it is apparent that there is some characteristic of each which we do not especially like and there is little we can do to prevent or counteract the undesirable feature without bringing a new element into the considerations. For this reason alone, we must weigh carefully every advantage and disadvantage these drug mixtures may present before using them indiscriminately in man. We reiterate that if intravenous mixtures such as are described above come into clinical use, we must remember that once injected they are irretrievable and one cannot as easily counteract an untoward response as is possible with an inhalation agent. It is also more difficult to know what antidote to use in a given case since we are always dealing with at least two drugs with rather different properties. ${ }^{1,8,9}$ Perhaps the most disconcerting problem that arises when such mixtures are employed in man is the possibility of psychoneurological reactions, especially when they occur several hours or days after the anaesthetic. ${ }^{8}$

Addiction is a problem that may only arise in a patient who needs repeated anaesthetics, and can be averted by changing the analgesic agent when subsequent operations are required. Extrapyramidal reactions and neuromuscular dyskinesias, although seldom recognized in animals, occur in man more often than some are willing to admit. Although these reactions can be controlled by drugs, the effect is sometimes frightening and the patient must be closely observed for at least one day after such an anaesthetic. In the case of the butyrophenones, such reactions are frequently delayed as much as two days. Hallucinations also occur with some of the potent analgesic drugs. Unfortunately, their occurrence cannot be studied in animals and it requires several days of close 
follow-up of psychic responses of the patient for them to be revealed. We are remiss in our duty in the care of our patients if this reaction is not sought out, for the long-term effect of such a reaction may be more incapacitating to the patient than the disease for which the surgical procedure and anaesthetic was given in the first place.

\section{SUMmaRy AND CoNClusions}

Mixtures of several analgesic and neuroleptic drugs were devised and used in sufficient dosage to provide smooth andesthesia in dogs. The response of the cardiorespiratory system, metabolic reactions, and postanaesthetic recovery were compared from recordings of the vital signs, blood analyses, and direct observation. These data show that the mixtures of anileridine-thiopental $(1: 10)$, fentanyl-droperidol (1:50), fentanyl-thiopental (1:1000), meperidine-thiopental (1:4), pentazocine-thiopental (1:33), and methotrimeprazine-thiopental (1:40) provide satisfactory anaesthetic conditions when used along with the inhalation of nitrous oxide and oxygen, and pulmonary ventilation is controlled during the period of anaesthesia. The thiopental-d'tubocurarine mixture would have been included if it had not caused severe post-anaesthetic respiratory depression. It is suggested that similar conditions may be reproduced clinically with all of these mixtures, but there are two major disadvantages to using such an anaesthetic technique: controllability is difficult to maintain and delayed reactions may be highly undesirable.

\section{RÉSUMÉ}

On a utilisé des mélanges de plusieurs médicaments analgésiques et neuroleptiques à des doses suffisantes pour produire une anesthésie légère chez des chiens. Les effets sur le système cardio-respiratoire, les réactions métaboliques et l'évolution postanesthésique ont été comparés d'après les tracés des signes vitaux, les analyses de sang et l'observation directe. Ces données montrent que les mélanges de anilérédine-thiopental (1:10), fentanyl-dropéridol (1:50), fentanylthiopental (1:1000), mépéridine-thiopental (1:4), pentazocine-thiopental (1:33), méthotriméprazine-thiopental $(1: 40)$ procurent des conditions anesthésiques satisfaisantes lorsqu'ils sont combinés à une inhallation de protoxyde d'azote et d'oxygène, et que la ventilation est contrôlée durant l'anesthésie. Nous aurions ajouté le mélange thiopental- $d$-tubocurarine, mais il produit une grave dépression respiratoire postanesthésique. On est d'avis que tous ces mélanges peuvent produire cliniquement de telles conditions, mais que cette technique d'anesthésie présente deux inconvénients sérieux: il est difficile de contrôler la ventilation et il peut se produire des réactions tardives indésirables.

\section{ACKNOWLEDGMENTS}

The authors are indebted to Ronald Welgan for technical assistance in the animal laboratory and to Mrs. Arckie Canton in the biochemistry laboratory. 
This work was supported by grants-in-aid from McNeil Laboratories, Inc. (Dr. E. C. Hessert), Winthrop-Sterling Research Institute (Dr. J. G. Bird), and Abbott Laboratories (Dr. N. Wheeler).

Methotrimeprazine was provided by The American Cyanamid CompanyLederle Division.

Pentazocine was provided by Winthrop-Sterling Research Institute.

Innovar, fentanyl, and droperidol were provided by McNeil Laboratories, Inc. Chlorprothixene was provided by Hoffmann-La Roche, Inc. (Dr. J. C. Howard, Ir.).

\section{REFERENCES}

1. Dobkin, A. B. Sedatives, Analgesics, Antidotes and Their Interaction: A Review. Canad. Anaesth. Soc. J. 11:252 (1964).

2. BAIRD, J. W. Pentothal-Curare Mixture. Anesthesiology 8: 75 (1947).

3. Hudon, F.; Jacques, A.; \& Borvan, P. A. Fluothane-Ether: An Azeotropic Mixture. Canad. Anaesth. Soc. J. 5: 403 (1958).

4. Geddes, I. C., \& Gray, T. C. Hyperventilation for Maintenance of Anaesthesia. Lancet ii: 4 (1959).

5. JANSsen, P. A. A Review of the Chemical Features Associated with Strong Morphine-like Activity. Brit. J. Anaesth. 34: 260 (1962).

6. Janssen, P. A.; Niemegeers, C. J.; Schellekens, K. H.; Verbruggen, F. I.; \& Van Neuten, J. M. The Pharmacology of Dehydrobenzperidol ( $R$ 4749), a New Potent and Short-Acting Neuroleptic Agent Chemically Related to Haloperidol. Arzneimittelforsch. 13: 205 (1963).

7. Holderness, M. C.; Chase, P. E.; \& Dripps, R. D. A Narcotic Analgesic and Butyrophenone with Nitrous Oxide for General Anesthesia. Anesthesiology 24: 336 (1963).

8. Dokmin, A. B.; Lee, P. K. Y.; Byles, P. H.; \& Israel, J. S. Neuroleptanalgesics: A Comparison of the Cardiovascular, Respiratory and Metabolic Effects of Innovan and Thiopentone Plus Methotrimeprazine Brit. J. Anaesth. 35: 694 (1963).

9. Dobrin, A. B.; Israel, J. S.; \& Brles, P. H. Innovan-N 2 O Anaesthesia in Normal Men: Effect on Respiration, Circulatory Dynamics, Liver Function, Metabolic Functions, Acid-Base Balance and Psychic Responses. Canad. Araesth. Soc. J. 11: 41 (1964).

10. Yelnosky, J.; Katz, R.; \& Dietrich, E. V. A Study of Some of the Pharmacologic Actions of Droperidol. Toxicol. Appl. Pharmacol. 6: 37 (1964).

11. Gardocki, J. F., \& Yelnosky, J. A Study of Some of the Pharmacologic Actions of Fentanyl Citrate. Toxicol. Appl. Pharmacol. 6: 48 (1964).

12. Yelnosky, J., \& GARDockI, J. F. A Study of Some of the Pharmacologic Actions of Fentanyl Citrate and Droperidol. Toxicol. Appl. Pharmacol. 6: 63 (1964).

13. Henscher, W. F. Principes et Technique de la Neuroleptanalgésie. Proc. XIIIeme Congrès Français d'Anesthésiologie, Bordeaux (1963).

14. Harris, L. S., \& Pierson, A. K. Some Narcotic Antagonists in Benzomorphan Series. J. Pharmacol. \& Exper. Therap. 143: 141 (1964).

15. Cass, L. J.; Frederik, W. S.; \& Teodono, J. V. Pentazocine as an Analgesic. Clinical Evaluation. T.A.M.A. 188: 112 (1964).

16. Lasagna, L.; DeKornfeld, T. J.; \&.Pearson, I. W. The Analgesic Efficacy and Respiratory Effects in Man of a Benzomorphan "Narcotic Antagonist." J. Pharmacol. \& Exper. Therap. 144: 12 (1964).

17. Fraser, H. F., \& Rosenburg, D. S. Studies on Human Addiction Liability of 2'-Hydroxy5,9-Dimethyl-2-(3,3-Dimethylallyl)-6,7-Benzomorphan (Win 20, 228): Weak Narcotic Antagonist. J. Pharmacol. \& Exper. Therap. 143: 149 (1964).

18. Courvoisier, S., \& Leau, O. Experimental Analgesic Activity of Levomepromazine. C. R. Acad. Sci. (Paris) 248: 3227 (1959).

19. IsBeLL, H., \& Wolbach, A. B. Observations on the Human Fharmacology and Addictiveness of Methotrimeprazine. Clin. Pharmacol. \& Therap. 4: 596 (1963).

20. Dobris, A. B., \& Purkin, N. Double Blind Study of Phenothiazines Used in Pre-anaesthetic Medication: A Clinical Evaluation of Promethazine (Phenergan), Promazine (Sparine), Prochlorperazine (Stemetil), and Levomepromazine (Nozinan). Canad. Anaesth. Soc. J. 7: 158 (1960). 
21. Pellmont, B.; Stenner, F. A.; Besendorf, H.; Baechtold, H. P.; \& LAeuppi, E. Zur Pharmakologie des Taractan, eines Neurolepticums mit besonderem Wirkungscharacter. Helv. physiol. pharmacol. Acta 18: 241 (1960).

22. Remvig, J., \& Sonne, L. M. Chlorprothixene (Truxal) Compared to Chlorpromazine. Psychopharmacologia (Berl.) 2: 203 (1961).

23. Dobkin, A. B.; IsRaei, J. S.; ByLes, P. H.; \& LeE, P. K. Y. Chlorprothixene and Amitriptyline: Interaction with Thiopentone, Circulatory Effect and Antisialogogue Effect. Brit. J. Anaesth. 35: 425 (1963).

24. DoвKIN, A. B. The Takaoka Respirator for Automatic Ventilation of the Lungs. Canad. Anaesth. Soc. J. 8: 556 (1961).

25. Byles, P. H. Observations on Some Continuously-Acting Spirometers. Brit. J. Anaesth. 32: 470 (1960).

26. Polgar, G., \& Forster, R. E. Measurement of Oxygen Tension in Unstirred Blood with a Platinum Electrode. I. Appl. Physiol. 15: 706 (1960).

27. Stow, R. W.; BAER, R. F.; \& RANDALL, B. F. Rapid Measurement of Tension of Carbon Dioxide in Blood. Arch. Phys. Med, 38: 346 (1957).

28. Severinghaus, J. W., \& Bradiey, A. F. Electrode for Blood $\mathbb{P}_{\mathrm{O}_{2}}$ and $\mathbb{P}_{\mathrm{CO}_{2}}$ Determination. J. Appl. Physiol. 13: 515 (1958).

29. NATELSON, S. Routine Use of Ultramicromethods in Clinical Laboratory. Amer. J. Clin. Pathol. 21: 1153 (1951).

30. Hoffman, W. S. A Rapid Photoelectric Method for the Determination of Glucose in Blood and Urine. J. Biol. Chem. 120: 51 (1937).

31. Marsh, W. H.; Fingerhut, B.; \& KIrSCH, E. Determination of Urea Nitrogen with the Diacetyl Method and an Automatic Dialysing Apparatus. Amer. J. Clin. Pathol. 28: 681 (1957).

32. Reitman, S., \& Franisel, S. A Colorimetric Method for the Determination of Serum Glutamic Oxalacetic and Glutamic Pyruvic Transaminases. Amer. I. Clin. Pathol. 28: 56 (1957).

33. Beecher, H. K. Measurement of Pain. Pharmacol. Rev. 9: 59 (1957).

34. Artusio, J. E., Jr., \& Van Posnar, A. The Concept of Intermediate Potency. Far East J. Anesth. 2: 37 (1958).

35. Gray, T. C. Disintegration of the Nervous System. Ann. Roy. Coll. Surg. Engl. 15: 402 (1954).

36. Laborit, H., \& Huguenard, P. Practique de l'Hibernothérapie en Chirurgie et en Médecine. Paris: Masson (1954).

37. Litrue, D. M., \& Stephen, C. R. Modem Balanced Anesthesia. Anesthesiology 15: 246 (1954).

38. Starr, I. The Use and Abuse of Mixtures of Active Drugs: Requirements of Modern Drug Therapy. J.A.M.A. 181: 106 (1962).

39. Delay, J., \& Denncer, P. Méthodes chimiothérapiques en psychiatrie: Les nouveaux médicaments psychotropes. Paris: Masson (1961).

40. DeCastro, G., \& Mundeleer, $\mathbb{P}$. Anesthésie sans barbituriques: La neuroleptanalgésie. Anesth. \& Analg. 16: 1022 (1959).

41. - - Neuroleptanalgésie: Definitions, principes, drogues, formules, avantages, problemes. Agressologie 3: 7 ( 1962).

42. Ninsson, E., \& JAnssen, $\mathbb{P}$. Neurolept-analgesia, an Alternative to General Anaesthesia. Acta Anaesth. Scandinav. 5: 73 (1961).

43. Nilsson, E. Origin and Rationale of Neurolept-Analgesia. Anesthesiology 24: 267 (1963).

44. Honsley, J. S. Narco-analysis. Lancet i: 55 (1936).

45. Jarman, R., \& ABEL, A. L. Intravenous Anaesthesia with Pentothal Sodium. Lancet i: $422(1936)$.

46. BAIRD, J. W. Pentothal-Curare Solution. Observations on Its Use in 500 Cases. Anesth. \& Analg. 27: 336 (1948).

47. Cohen, E. N.; PAUlson, W. J.; Wall, J.; \& ElLert, B. Thiopental, Curare, and Nitrous Oxide Anaesthesia for Cesaerean Section with Studies on Placental Transmission. Surg., Gynec. \& Obst. 97: 456 (1953).

48. Gray, T. C., \& RIDING, J. E. Anaesthesia for Mitral Valvulotomy. Anaesthesia 12: 129 (1957). 